\title{
Reduction of Cellular Lipid Content by a Knockdown of Drosophila PDP1 $\gamma$ and Mammalian Hepatic Leukemia Factor
}

\author{
Svetlana Dzitoyeva and Hari Manev \\ Department of Psychiatry, Psychiatric Institute, University of Illinois at Chicago, Chicago, IL 60612, USA \\ Correspondence should be addressed to Svetlana Dzitoyeva; sdzitoyeva@psych.uic.edu
}

Received 22 March 2013; Accepted 19 July 2013

Academic Editor: Philip W. Wertz

Copyright (c) 2013 S. Dzitoyeva and H. Manev. This is an open access article distributed under the Creative Commons Attribution License, which permits unrestricted use, distribution, and reproduction in any medium, provided the original work is properly cited.

In exploring the utility of double-stranded RNA (dsRNA) injections for silencing the PAR-domain protein 1 (Pdp1) gene in adult Drosophila, we noticed a dramatic loss of fat tissue lipids. To verify that our RNAi approach produced the expected Pdp1 knockdown, the abdominal fat tissues sections were stained with PDP1 antibodies. PDP1 protein immunostaining was absent in flies injected with dsRNA targeting a sequence common to all known $P d p 1$ isoforms. Subsequent experiments revealed that lipid staining is reduced in flies injected with dsRNA against Pdpl $\gamma$ (fat body specific) and not against Pdple (predominantly involved in circadian mechanisms). Drosophila PDPl $\gamma$ protein shows a high homology to mammalian thyrotroph embryonic factor (TEF), albumin D site-binding protein (DBP), and hepatic leukemia factor (HLF) transcription factors. In an in vitro model of drug- (olanzapine-) induced adiposity in mouse 3T3-L1 cells, the mRNA content of HLF but not TEF and DBP was increased by the drug treatment. A knockdown of the HLF mRNA by transfecting the cultures with HLF dsRNA significantly reduced their lipid content. Furthermore, the HLF RNAi prevented olanzapine from increasing the cell lipid content. These results suggest that the PDP1/HLF system may play a role in physiological and drug-influenced lipid regulation.

\section{Introduction}

Contrary to the previous belief that in adult Drosophila (fruit fly) RNA interference (RNAi) cannot occur by exogenous administration of double-stranded RNA (dsRNA), it was conclusively demonstrated that this type of systemic RNAi is operative in adult flies [1]. This mechanism explains the efficacy of dsRNA injections into adult Drosophila, applied as a tool for targeted gene knockdown in adult organisms [2-4]. The advantage of this RNAi method is that it avoids the unwanted developmental alterations and the possible side effects of genetic manipulations involved in alternative RNAi approaches. In the course of exploring the utility of dsRNA injections for silencing the PAR-domain protein 1 (Pdp1) gene in adult Drosophila, we noticed a peculiar RNAi-related phenotype, a dramatic loss of fat tissue lipids. Here, we report the follow-up study aimed at exploring this serendipitous discovery.

Drosophila Pdp1 encodes a transcription factor highly homologous to the proline- and acidic amino acid-rich (PAR) subfamily of mammalian bZIP transcription factors, albumin D site-binding protein (DBP), hepatic leukemia factor (HLF), and thyrotroph embryonic factor (TEF). PDP1 was originally identified as a regulator of the muscle activator region [5]. Subsequently, it was established that $P d p 1$ is a component of the Drosophila circadian network and that its expression is directly activated by $d$ Clock/Cycle genes $[6,7]$. The $P d p 1$ gene encodes multiple transcripts, which are differentially expressed during embryogenesis [8]. Of the six Pdp1 isoforms, $P d p 1 \varepsilon$ is the one that shows a circadian expression and is involved in the regulation of the Drosophila circadian behavior [9]. Whereas Pdple is predominantly expressed in the nervous system, $P d p l \gamma$ is predominantly expressed in the fat body [8]. The mammalian homologous factors DBP, TEF, and HLF also show a circadian rhythm in their expression/accumulation; their absence results in epilepsy [10]. Furthermore, these mammalian proteins have recently been linked to a mechanism of fatty acid regulation [11]. To address possible similarities between Drosophila Pdp1 and its mammalian homologues, in addition to the experiments in 
TABLE 1: Primers used for the mRNA assay.

\begin{tabular}{|c|c|c|}
\hline Target & Forward & Reverse \\
\hline HLF & $5^{\prime}$-gaaggagctgggcaaatgcaagaa- $3^{\prime}$ & $5^{\prime}$-accagacaggaaacaagctgtcca-3' \\
\hline Cyclophilin & $5^{\prime}$-agcatacaggtcctggcatcttgt- $3^{\prime}$ & $5^{\prime}$-aaacgctccatggcttccacaatg- $3^{\prime}$ \\
\hline Pdp1 total & $5^{\prime}$-acttccctcctcagttgccgg- $3^{\prime}$ & $5^{\prime}$-tcgcagatggtctgtgtgta- $3^{\prime}$ \\
\hline Pdple & $5^{\prime}$-ccacgctaacgtaccagaggattt- $3^{\prime}$ & $5^{\prime}$-ctgaaatcgcgctttcaagctgtt- $3^{\prime}$ \\
\hline $\operatorname{Pdpl} \gamma$ & $5^{\prime}$-gagtttcagttacgcgttgttgct- $3^{\prime}$ & $5^{\prime}$-gttgttctccgacaggaactcgtc- $5^{\prime}$ \\
\hline RP49 & $5^{\prime}$-atgaccatccgcccagcataca- $3^{\prime}$ & $5^{\prime}$-tgtgtattccgaccaggttac- $3^{\prime}$ \\
\hline
\end{tabular}

fruit flies, we employed a model of drug-induced adiposity in mammalian 3T3-L1 cells in vitro [12].

\section{Material and Methods}

2.1. Drosophila and Cell Culture. Male Canton-S flies were cultured for 5-7 days in a $12 \mathrm{~h} / 12 \mathrm{~h}$ light/dark cycle [3]. They were $\mathrm{CO}_{2}$ anesthetized, injected with 0.3-0.4 pmol dsRNA [3], and collected five days later. Mouse 3T3-L1 cells (American Type Culture Collection), grown to confluence in Dulbecco's modified Eagle's medium (GIBCO) $/ 10 \%$ fetal bovine serum (Atlanta Biologicals), were differentiated into adipocytes by changing to a differentiation medium [12]. dsRNA transfection (10 pmol; TransPass R1 transfection reagent; New England Biolabs) started $24 \mathrm{~h}$ after addition of the differentiation medium; cells were collected $48 \mathrm{~h}$ later. Olanzapine (50 $\mu \mathrm{M}$; Sequoia Research Products Ltd.) and vehicle (1:1000 dimethyl sulfoxide; Sigma) were added either together with the differentiation medium or $24 \mathrm{~h}$ after the initiation of dsRNA transfection.

2.2. Double-Stranded RNA (dsRNA). For Drosophila dsRNA RNAi, the corresponding RNAs were synthesized in vitro from DNA oligonucleotides with an attached T7 RNA polymerase promoter sequence (IDT DNA Technologies) targeting the aacttccctcctcagttgccgg cDNA region common to all Pdp1 isoforms (AF172402, AF172403, AF172404, AF172405, AF172406, and AF209903), the agttacgcgttgttgctgcgacc cDNA region unique to Pdpl $\gamma$ (AF172404), and the aacgtaccagaggatttaccagg region unique to $P d p 1 \varepsilon$ (AF172406). Control dsRNA was based on the human 5-lipoxygenase cDNA, ttcatgcacatgttccagtctt (NM_000698; no matches to Drosophila genome sequences; Drosophila does not have 5lipoxygenase homologues). The single-stranded sense and antisense DNA oligonucleotides were annealed in distilled water and $100 \mathrm{ng}$ was used in $30 \mu \mathrm{L}$ of in vitro transcription reaction. dsRNA molecules were formed by combining the corresponding transcribed sense and antisense RNAs $\left(10 \mathrm{~min}\right.$ at $\left.65^{\circ} \mathrm{C}\right)[2,3]$. For mouse $3 \mathrm{~T} 3-\mathrm{L} 1 \mathrm{dsRNA}$ RNAi, a 154 nucleotide fragment (1120-1274) of mouse HLF mRNA (NM_172563) was cloned into a pGEM-T vector (Promega). The insert was amplified with M13 primers and RNA was transcribed in vitro with T7 and SP6 RNA polymerases (New England Biolabs). An equal amount of the single-stranded sense and antisense RNA molecules was incubated for $10 \mathrm{~min}$ at $65^{\circ} \mathrm{C}$ and cooled on ice. Control dsRNA was prepared from a 239-nucleotide fragment (394-620) of the green fluorescent protein gene (X83959) amplified from the pGFP cloning plasmid DNA vector.

2.3. $m R N A$ Assay. RNA was extracted from cells and Drosophila homogenates with TRIzol reagent (Invitrogen), reverse transcribed, and used for the quantitative real time PCR (Stratagene Mx3005P qPCR System; Agilent Technologies with Maxima SYBR Green qPCR Master Mix (MM); Fermentas Inc.), in a two-step PCR program [14]: the reaction mix: $10 \mu \mathrm{L} 2 \mathrm{x}$ MM, $2 \mu \mathrm{L}$ primer $\operatorname{mix}(0.2 \mu \mathrm{M}$ final, each), and $8 \mu \mathrm{L}$ reverse transcription mix. The corresponding primers are shown in Table 1. Data were normalized against the cyclophilin (3T3-L1 cells) or RP49 (Drosophila) internal control and presented as a coefficient of variation [14].

2.4. PDP1 Immunostaining. 30-micron Drosophila cryosections were fixed in $12 \%$ formaldehyde/phosphate buffered saline (PBS), rinsed in PBS, and incubated with PDP1 antibody $\left(1: 500\right.$, overnight, $\left.4^{\circ} \mathrm{C}\right)$ [8] followed by incubation with secondary biotinylated anti-rabbit IgG ( $1 \mathrm{~h}$; Vector Laboratories). Color was developed with DAB (Sigma).

2.5. Lipid Staining and Quantification. For lipid visualization in the Drosophila fat body, whole fly 30 -micron cryosections were fixed, incubated with Oil Red O (ORO; Sigma; 0.5\% in isopropanol diluted with water $3: 2$ and filtered through a $0.45 \mu \mathrm{m}$ filter) for $30 \mathrm{~min}$, rinsed, covered with $80 \%$ glycerol, and photographed. For quantitative measurements, flies were decapitated and their bodies cryosectioned and fixed. Freefloating sections (from 5 bodies/data point) were stained with ORO, washed, and dried, and the dye was recovered with isopropanol. 3T3-L1 cells were fixed and incubated (30 min) with ORO. After discarding this solution, the dye captured by intracellular lipids was recovered with $400 \mu \mathrm{L}$ isopropanol, and the absorbance was measured (single wavelength, $520 \mathrm{~nm}$ filter; Bio-Rad, Model 550). The results are reported in units.

2.6. Statistics. Statistical analyses were performed using SPSS software (SPSS Inc.). Data (mean \pm SEM) were analyzed by one-way analysis of variance followed by Student's $t$-test or Dunnett's test (significance at $P<0.05$ ).

\section{Results}

3.1. Drosophila Pdp1 dsRNA RNAi. Our initial observation of the lipid-reducing effect of $P d p 1$ RNAi was made by examining fly body sections without the help of any lipid 


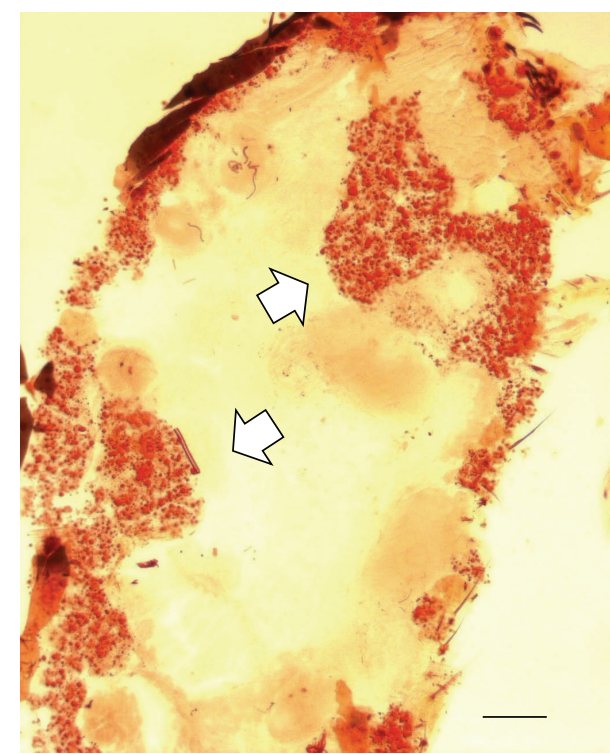

(a)

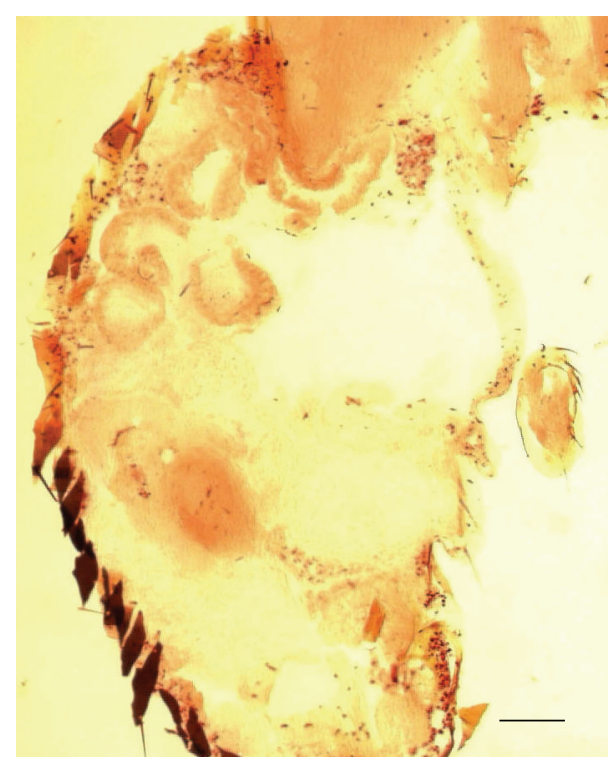

(b)

FIgURE 1: Effect of total Pdp1 RNAi induced in adult Drosophila on lipid staining. Flies were injected with control dsRNA (panel (a)) and dsRNA targeted at a sequence common to all known $P d p 1$ isoforms (total $P d p 1$ RNAi; panel (b)). They were processed for lipid staining (red) five days later. Shown is a low magnification (objective $5 \mathrm{x}$ ) sagittal abdominal section ( in the fat tissue cells of a control fly (indicated by white arrows) (a) and its absence in the Pdp1 RNAi fly (b).

staining; that is, the effect was rather obvious. To verify this observation, we employed the ORO lipid staining. In these experiments, a total $P d p 1$ RNAi was achieved by injecting flies with the dsRNA targeted at a sequence common to all known $P d p 1$ isoforms. This resulted in the loss of ORO lipid staining as exemplified by the staining of Drosophila abdominal sections (Figure 1). To verify that our RNAi approach produced the expected Pdp1 knockdown, we stained the whole fly sections including the abdominal fat tissue with PDP1 protein antibodies. PDP1 immunostaining revealed a robust nuclear PDP1 staining in the cells of flies injected with control dsRNA and the absence of PDP1 staining in Pdp1 dsRNA injected flies (Figure 2). Also, in these sections, we confirmed the lipidreducing effect of the total Pdp1 dsRNA; the number and the size of lipid-containing droplets in fat tissue cells were reduced in the RNAi samples (Figure 2).

The previous results were obtained with the dsRNA targeted at a sequence common to all known $P d p 1$ isoforms. In subsequent experiments, we investigated the $P d p 1$ isoform specificity of the lipid-reducing phenotype. Hence, flies were injected with dsRNA targeted specifically against $P d p 1 \varepsilon$ (predominantly present in the nervous system and involved in circadian mechanisms) and $P d p 1 \gamma$ (expressed in the fat body), respectively, and their body lipid content was quantified. Only the $P d p 1 \gamma$ dsRNA significantly reduced the body lipid content (Figure 3).

3.2. Mammalian HLF dsRNA RNAi. Drosophila PDPl $\gamma$ protein shows a high homology to TEF, DBP, and HLF members of the PAR subfamily of mammalian bZIP transcription factors (Figure 4) [13]. To explore possible similarities between
Pdpl $\gamma$ and these factors, we selected the mouse 3T3-L1 preadipocytes in vitro. These cells have been used as a model for drug-induced adipogenic effects; that is, treatment of 3T3-L1 cells during differentiation into adipocytes with the antipsychotic drug olanzapine increases their lipid content [12]. In this model, we found that the adipogenic olanzapine treatment increases the mRNA content of HLF but not TEF and DBP (Figure 5). On the other hand, a knockdown of the endogenous HLF mRNA by transfecting the cultures with HLF dsRNA significantly reduced their lipid content (Figure 6). In an experiment in which olanzapine and vehicle treatments were initiated $24 \mathrm{~h}$ after the initiation of transfection and conducted for the next $24 \mathrm{~h}$, we found that olanzapine treatment increased lipid content in naïve and sham-dsRNA transfected cells but not in HLF dsRNAtransfected cells (Figure 7).

\section{Discussion}

In this work, we confirmed and expanded our serendipitous observation that a systemic PDP1 knockdown in adult flies, induced by injections of $P d p 1$ dsRNAs, leads to a significant lipid decrease, and we found that similar phenotype can be induced by HLF RNAi in mouse adipocytes in vitro. Collectively, our results demonstrated that a reduction of the PDP $1 \gamma /$ HLF transcription factor leads to a decreased lipid content.

Both mammalian HLF and Drosophila PDP1 are known components, that is, output regulators, of circadian cycles and as such have been linked to metabolic regulation [15]. The expression of Drosophila circadian genes (i.e., peripheral clocks) in the fat body has been shown to play a role in the 


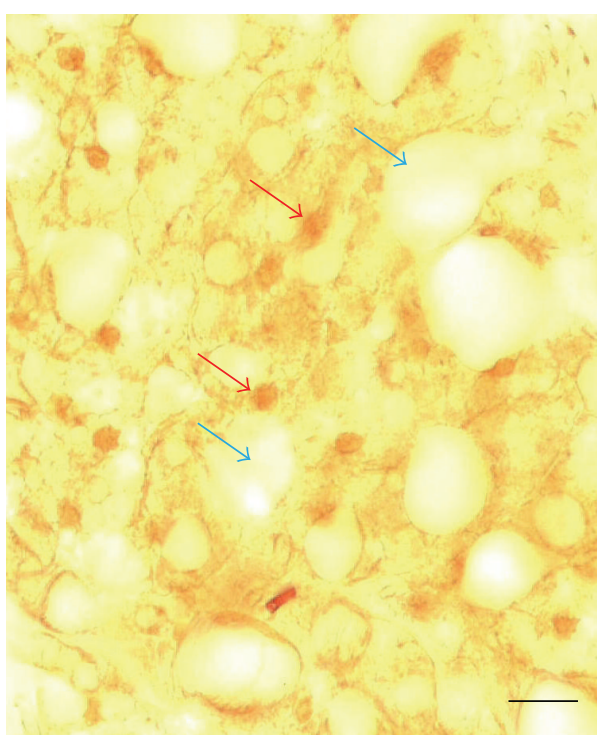

(a)

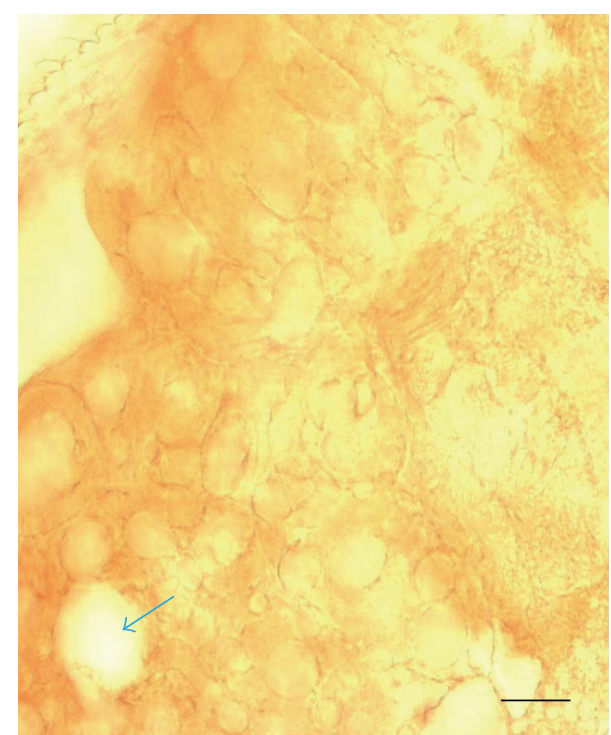

(b)

Figure 2: Effect of total Pdp1 RNAi induced in adult Drosophila on PDP1 protein immunostaining in the fat tissue. Flies were injected with control dsRNA (panel (a)) and dsRNA targeted at a sequence common to all known Pdp1 isoforms (total Pdp1 RNAi; panel (b)). They were processed for PDP1 immunolabeling five days later. Shown is a high magnification (objective 20X) of abdominal fat tissue section (size bar = $20 \mu \mathrm{m}$ ). Note the presence of strong nuclear PDP1 protein immunolabeling (dark circles indicated by red arrows) in the control section (a) and its absence in the Pdp1 RNAi section (b). Also, indicated (blue arrows) are multiple white circles of lipid droplet-containing cells in the control section and their reduced number after Pdp1 RNAi.



FIGURE 3: Quantitative assay of Drosophila lipid content following the isoform-specific Pdp1 RNAi. Adult flies were injected with control, $P d p 1 \gamma$, and $P d p 1 \varepsilon$ dsRNA and processed for quantitative lipid assay. $P d p 1 \gamma$ but not $P d p 1 \varepsilon$ dsRNA reduced body lipid content $\left({ }^{*} P<0.001\right.$ versus control; $n=5$; mean \pm standard error mean).

regulation of fly metabolism [16]. Of the six $P d p 1$ isoforms, $P d p 1 \varepsilon$ is the one that is characterized by a prominent circadian expression and is involved in the regulation of the Drosophila circadian behavior [9]. One of the circadian functions of $P d p 1 \varepsilon$ is in regulating the circadian output gene takeout [17]. In male Drosophila, takeout is abundant in the fat body and plays a role in courtship behavior of these flies [18]. It was suggested that $P d p l \varepsilon$-mediated regulation of the fat body genes may influence this type of fly behavior [17]. In our Drosophila experiments, lipid staining was decreased by $P d p l \gamma$ and not Pdple RNAi. Hence, it would be interesting to elucidate whether in addition to $P d p 1 \varepsilon$ also $P d p 1 \gamma$ regulates the expression of the output genes such as takeout and whether this mechanism mediates the observed lipiddecreasing effects of $P d p 1 \gamma$ knockdown.

Our experiments with adipocytes show that in addition to systemic PDP1/HLF alterations (e.g., systemic PDP1 knockdown in adult flies), also direct cellular HLF alterations (e.g., knockdown in 3T3-L1 cells) can reduce lipid content.

In our in vitro experiments, a drug-induced adiposity was accompanied by increased levels of HLF mRNA, whereas HLF RNAi was accompanied by decreased lipid content. Furthermore, it was previously reported that in a mouse model of severe reduction of lipid accumulation and severe loss of body weight, the liver HLF mRNA levels, along with the TEF and DBP mRNA levels, are significantly reduced [19]. Hence, HLF appears to be involved in the physiological regulation of cellular lipid levels.

In our experimental conditions, the RNAi-mediated HLF mRNA reduction significantly diminished a drug -induced adiposity (i.e., olanzapine). Olanzapine belongs to a class of drugs known as the second generation antipsychotic drugs (SGADs). All these compounds are capable of triggering significant weight gain associated with adverse metabolic alterations [20, 21]. It has been proposed that these side effects may occur by a direct stimulatory action of SGADs on adipocytes $[12,22,23]$. Our in vitro experiments confirmed the direct adipogenic action of olanzapine and found that this action can be diminished by HLF reduction. In 


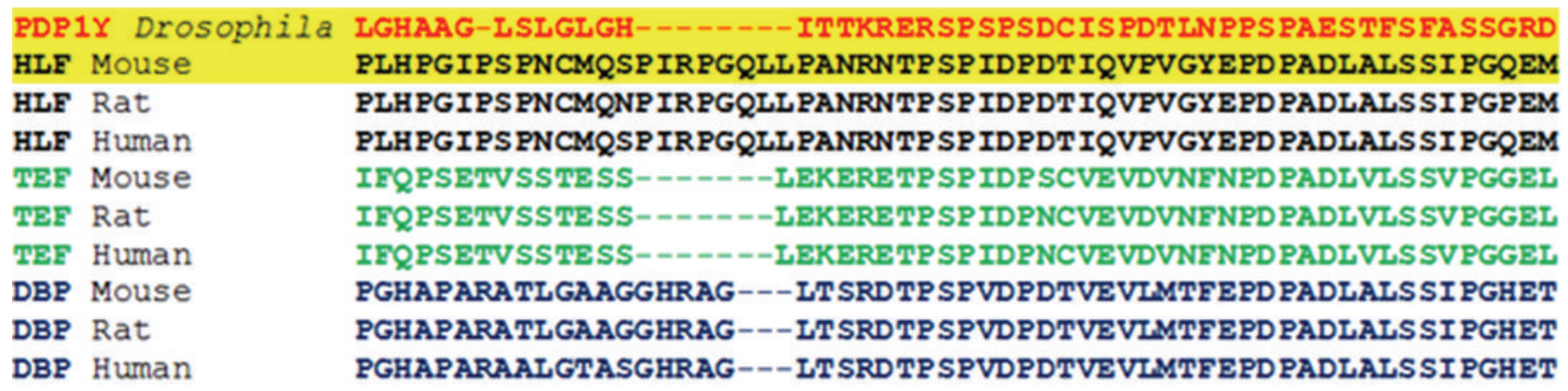

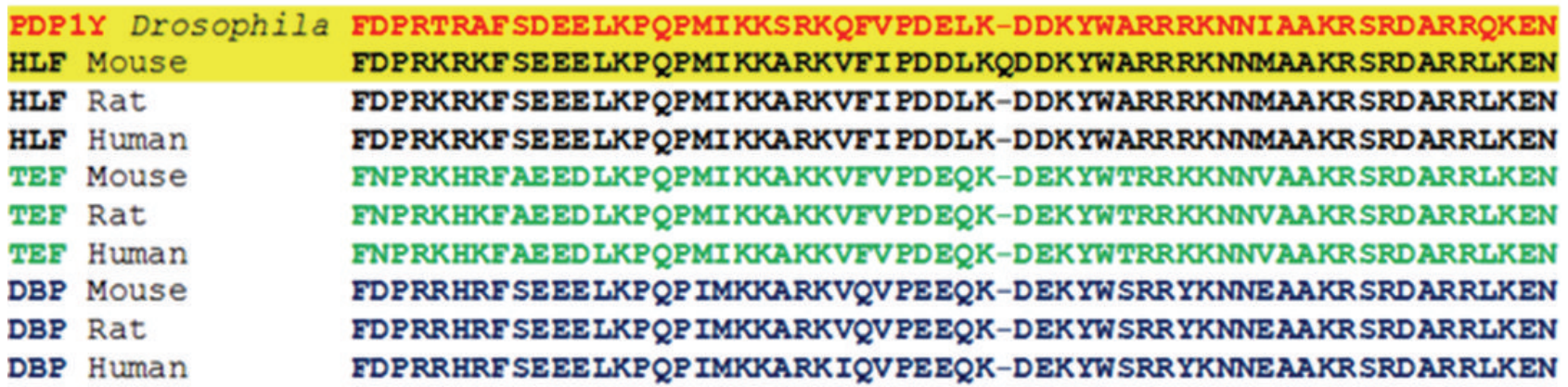

$\begin{array}{ll}\text { PDP1Y Drosophila } & \text { QIAMRARYLEKENATLHQEVEQLKQENMILIRLSKEQDV---- } \\ \text { HLF Mouse } & \text { QIAIRASFLEKENSALRQEVADLRKELGKCKNILAKYEARHGPL } \\ \text { HLF Rat } & \text { QIAIRASFLEKENSALRQEVADIRKELGKCKNILAKYEARHGPL } \\ \text { HLF Human } & \text { QIAIRASFLEKENSALRQEVADLRKELGKCKNILAKYEARHGPL } \\ \text { TEF Mouse } & \text { QITIRAAFLEKENTALRTEVAELRKEVGKCKTIVSKYETKYGPL } \\ \text { TEF Rat } & \text { QITIRAAFLEKENTALRTEVAELRKEVGKCKTIVSKYETKYGPL } \\ \text { TEF Human } & \text { QITIRAAFLEKENTALRTEVAELRKEVGKCKTIVSKYETKYGPL } \\ \text { DBP Mouse } & \text { QISVRAAFLEKENALLRQEVVAVRQELSHYRAVLSRYQAQHGTL } \\ \text { DBP Rat } & \text { QISVRAAFLEKENALLRQEVVAVQELSHYRAVLSRYQAQHGTL } \\ \text { DBP Human } & \text { QISVRAAFLEKENALLRQEVVAVRQELSHYRAVLSRYQAQHGAL }\end{array}$

FIgURE 4: Homology between Drosophila PDP1 $\gamma$ and mammalian bZIP transcription factors HLF, TEF, and DBP. Shown is the protein sequence alignment in the b-ZIP structural regions with the highest homology, analyzed using the online CLUSTALW multiple sequences alignment tool [13]. The NCBI database accession information is as follows. Drosophila PDP1 $\gamma$ Q9TVQ4; HLF: mouse NP_766151, rat Q64709, and human NP_002117; TEF: mouse NP_059072, rat NP_062067, and human NP_003207; DBP: mouse NP_058670, rat NP_036675, and human NP_001343. Highlighted in yellow are the sequences of Drosophila PDP1 $\gamma$ and mouse HLF.

the therapy of psychiatric patients with SGADs, a better understanding of the mechanisms that lead to metabolic side effects is needed to identify the risk factors that facilitate and exacerbate this SGADs-associated clinical problem. Our results suggest for the first time that the HLF pathway could be such a mechanism. Furthermore, the observed direct susceptibility of the adipocyte HLF and lipids to regulation by drugs (e.g., olanzapine-increased HLF mRNA and lipid contents) suggests that future pharmacological tools could be tailored specifically to the adipocyte HLF pathway to interfere therapeutically with the mechanisms of adiposity.

\section{Abbreviations}

DBP: Albumin D site-binding protein

dsRNA: Double-stranded RNA
HLF: Hepatic leukemia factor

PAR: Proline- and acidic amino acid rich

PDP1: PAR-domain protein 1

RNAi: RNA interference

SGADs: Second generation antipsychotic drugs

TEF: Thyrotroph embryonic factor.

\section{Conflict of Interests}

The authors declare that they have no conflict of interests.

\section{Acknowledgments}

This work was supported by the Psychiatric Institute, University of Illinois at Chicago. The authors thank Nikola 




(a)



(b)

Figure 5: Effect of olanzapine on lipid content (a) and mRNA content of mammalian Pdp1 homologues (b) in mouse 3T3-L1 cells. Vehicle (open bars) and olanzapine ( $50 \mu \mathrm{M}$; closed bars) were present in the culture medium for $24 \mathrm{~h}$. Olanzapine increased lipid content (a) and HLF mRNA content (normalized to internal control mRNA) (b) $\left({ }^{*} P<0.001\right.$ versus corresponding control; $n=6$; mean \pm standard error mean).

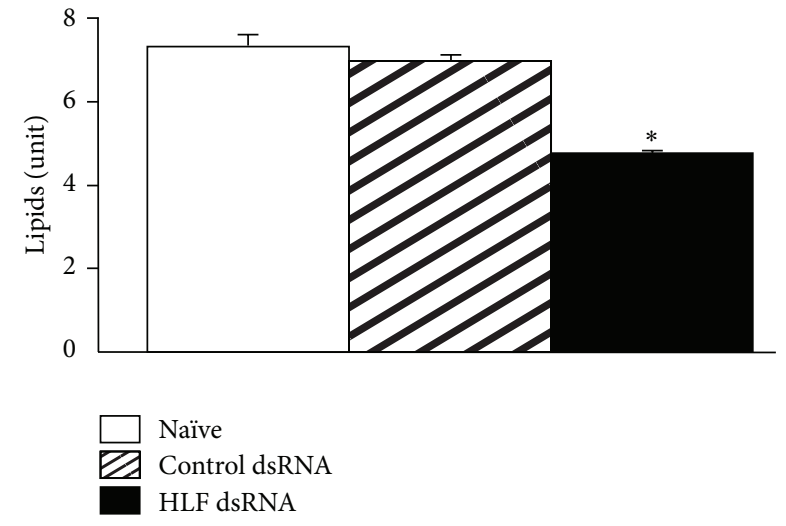

FIGURE 6: Effect of mammalian HLF RNAi on lipid content in mouse 3T3-L1 cells. Cells in culture were transfected with control and HLF dsRNA and processed for lipid assay $48 \mathrm{~h}$ later (see text for details). Lipid content in 3T3-L1 cells was reduced by HLF dsRNA treatment $\left({ }^{*} P<0.001\right.$ versus naïve; $n=6$; mean \pm standard error mean), which also reduced HLF mRNA content (not shown).

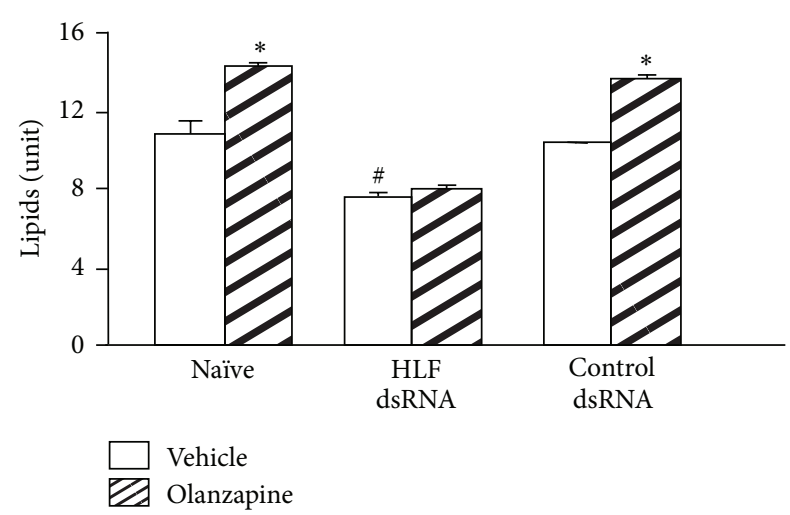

FIGURE 7: HLF RNAi prevents the adipogenic effect of olanzapine in mouse 3T3-L1 cells. Olanzapine and vehicle treatments were initiated $24 \mathrm{~h}$ after the initiation of transfection and conducted for the next $24 \mathrm{~h}$. At the end of the treatment, cells were assayed for lipid content $\left({ }^{*} P<0.001\right.$ versus corresponding vehicle; ${ }^{\#} P<0.001$ versus naïve and control dsRNA transfection; $n=3$; mean \pm standard error mean).
Dimitrijevic for technical help, Robert V. Storti for PDP1 antibodies, and the late Erminio Costa, Director of the Psychiatric Institute, for support.

\section{References}

[1] M.-C. Saleh, M. Tassetto, R. P. Van Rij et al., "Antiviral immunity in Drosophila requires systemic RNA interference spread," Nature, vol. 458, no. 7236, pp. 346-350, 2009.

[2] S. Dzitoyeva, N. Dimitrijevic, and H. Manev, "Intra-abdominal injection of double-stranded RNA into anesthetized adult Drosophila triggers RNA interference in the central nervous system," Molecular Psychiatry, vol. 6, no. 6, pp. 665-670, 2001.

[3] S. Dzitoyeva, N. Dimitrijevic, and H. Manev, " $\gamma$-aminobutyric acid $B$ receptor 1 mediates behavior-impairing actions of alcohol in Drosophila: adult RNA interference and pharmacological evidence," Proceedings of the National Academy of Sciences of the United States of America, vol. 100, no. 9, pp. 5485-5490, 2003.

[4] A. Goto, S. Blandin, J. Royet, J.-M. Reichhart, and E. A. Levashina, "Silencing of Toll pathway components by direct injection of double-stranded RNA into Drosophila adult flies," Nucleic Acids Research, vol. 31, no. 22, pp. 6619-6623, 2003.

[5] S.-C. Lin, M.-H. Lin, P. Horváth, K. L. Reddy, and R. V. Storti, "PDP1, a novel Drosophila PAR domain bZIP transcription factor expressed in developing mesoderm, endoderm and ectoderm, is a transcriptional regulator of somatic muscle genes," Development, vol. 124, no. 22, pp. 4685-4696, 1997.

[6] S. A. Cyran, A. M. Buchsbaum, K. L. Reddy et al., "vrille, Pdp1, and dClock form a second feedback loop in the Drosophila circadian clock," Cell, vol. 112, no. 3, pp. 329-341, 2003.

[7] C. Hermann, R. Saccon, P. R. Senthilan et al., "The circadian clock network in the brain of different Drosophila species," Journal of Comparative Neurology, vol. 521, no. 2, pp. 367-388, 2013.

[8] K. L. Reddy, A. Wohlwill, S. Dzitoeva, M.-H. Lin, S. Holbrook, and R. V. Storti, "The Drosophila PAR domain protein 1 (Pdp1) gene encodes multiple differentially expressed mRNAs and proteins through the use of multiple enhancers and promoters," Developmental Biology, vol. 224, no. 2, pp. 401-414, 2000.

[9] C. Lim, J. Lee, E. Koo, and J. Choe, "Targeted inhibition of Pdple abolishes the circadian behavior of Drosophila melanogaster," 
Biochemical and Biophysical Research Communications, vol. 364, no. 2, pp. 294-300, 2007.

[10] F. Gachon, P. Fonjallaz, F. Damiola et al., "The loss of circadian PAR bZip transcription factors results in epilepsy," Genes and Development, vol. 18, no. 12, pp. 1397-1412, 2004.

[11] F. Gachon, N. Leuenberger, T. Claudel et al., "Proline- and acidic amino acid-rich basic leucine zipper proteins modulate peroxisome proliferator-activated receptor $\alpha$ (PPAR $\alpha$ ) activity," Proceedings of the National Academy of Sciences of the United States of America, vol. 108, no. 12, pp. 4794-4799, 2011.

[12] L.-H. Yang, T.-M. Chen, S.-T. Yu, and Y.-H. Chen, "Olanzapine induces SREBP-1-related adipogenesis in 3T3-L1 cells," Pharmacological Research, vol. 56, no. 3, pp. 202-208, 2007.

[13] C. Combet, C. Blanchet, C. Geourjon, and G. Deléage, “NPS@: network protein sequence analysis," Trends in Biochemical Sciences, vol. 25, no. 3, pp. 147-150, 2000.

[14] M. Imbesi, S. Dzitoyeva, L. W. Ng, and H. Manev, "5Lipoxygenase and epigenetic DNA methylation in aging cultures of cerebellar granule cells," Neuroscience, vol. 164, no. 4, pp. 1531-1537, 2009.

[15] G. Asher and U. Schibler, "Crosstalk between components of circadian and metabolic cycles in mammals," Cell Metabolism, vol. 13, no. 2, pp. 125-137, 2011.

[16] K. Xu, X. Zheng, and A. Sehgal, "Regulation of feeding and metabolism by neuronal and peripheral clocks in Drosophila," Cell Metabolism, vol. 8, no. 4, pp. 289-300, 2008.

[17] J. Benito, V. Hoxha, C. Lama et al., "The circadian output gene takeout is regulated by Pdple," Proceedings of the National Academy of Sciences of the United States of America, vol. 107, no. 6, pp. 2544-2549, 2010.

[18] B. Dauwalder, S. Tsujimoto, J. Moss, and W. Mattox, "The Drosophila takeout gene is regulated by the somatic sexdetermination pathway and affects male courtship behavior," Genes and Development, vol. 16, no. 22, pp. 2879-2892, 2002.

[19] M. T. Flowers, M. P. Keller, Y. Choi et al., "Liver gene expression analysis reveals endoplasmic reticulum stress and metabolic dysfunction in SCD1-deficient mice fed a very low-fat diet," Physiological Genomics, vol. 33, no. 3, pp. 361-372, 2008.

[20] D. B. Allison, J. W. Newcomer, A. L. Dunn et al., "Obesity among those with mental disorders: a National Institute of Mental Health meeting report," American Journal of Preventive Medicine, vol. 36, no. 4, pp. 341-350, 2009.

[21] R. Coccurello and A. Moles, "Potential mechanisms of atypical antipsychotic-induced metabolic derangement: clues for understanding obesity and novel drug design," Pharmacology and Therapeutics, vol. 127, no. 3, pp. 210-251, 2010.

[22] J. Minet-Ringuet, P. C. Even, P. Valet et al., "Alterations of lipid metabolism and gene expression in rat adipocytes during chronic olanzapine treatment," Molecular Psychiatry, vol. 12, no. 6, pp. 562-571, 2007.

[23] Z. Yang, J.-Y. Yin, Z.-C. Gong et al., "Evidence for an effect of clozapine on the regulation of fat-cell derived factors," Clinica Chimica Acta, vol. 408, no. 1-2, pp. 98-104, 2009. 

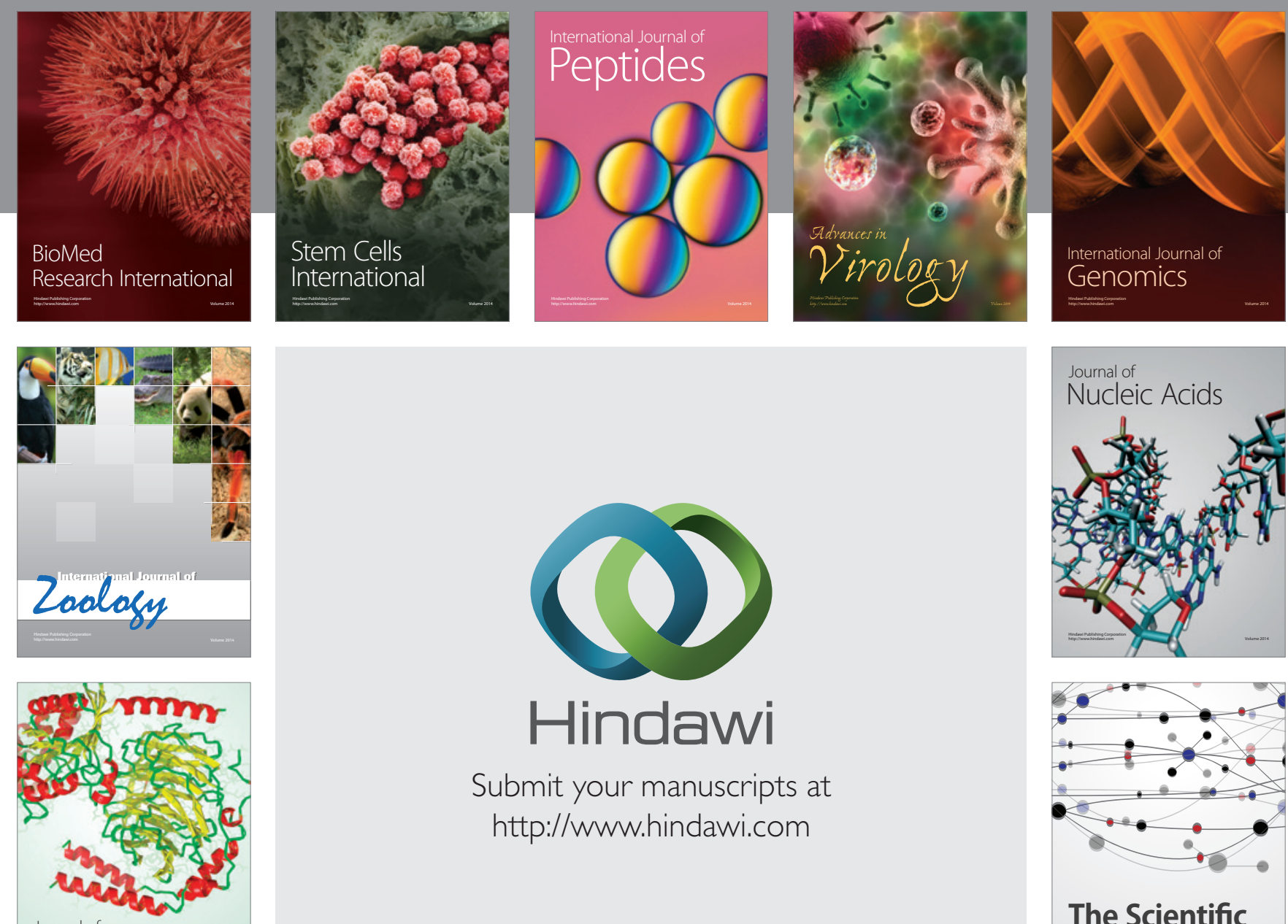

Submit your manuscripts at

http://www.hindawi.com

Journal of
Signal Transduction
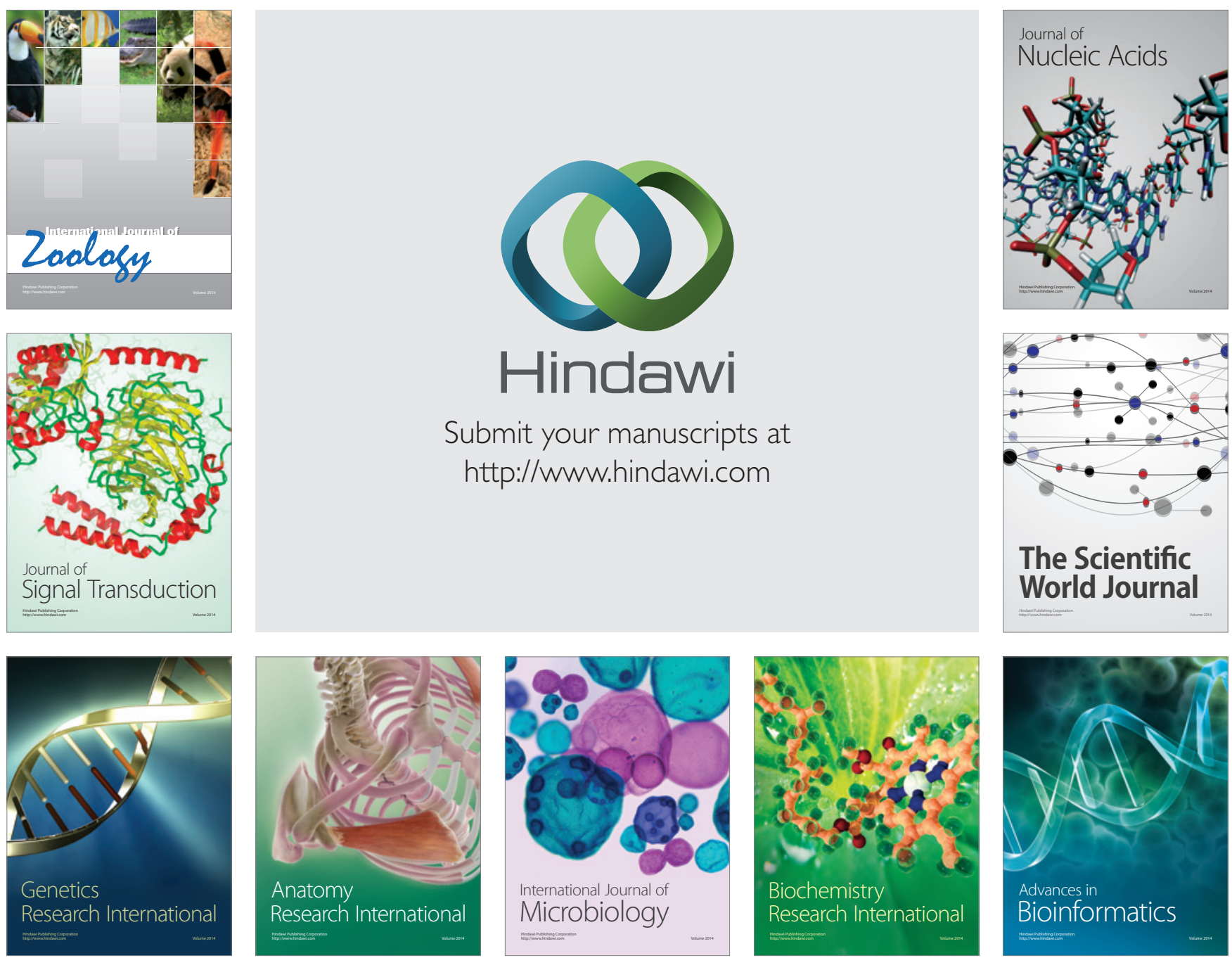

The Scientific World Journal
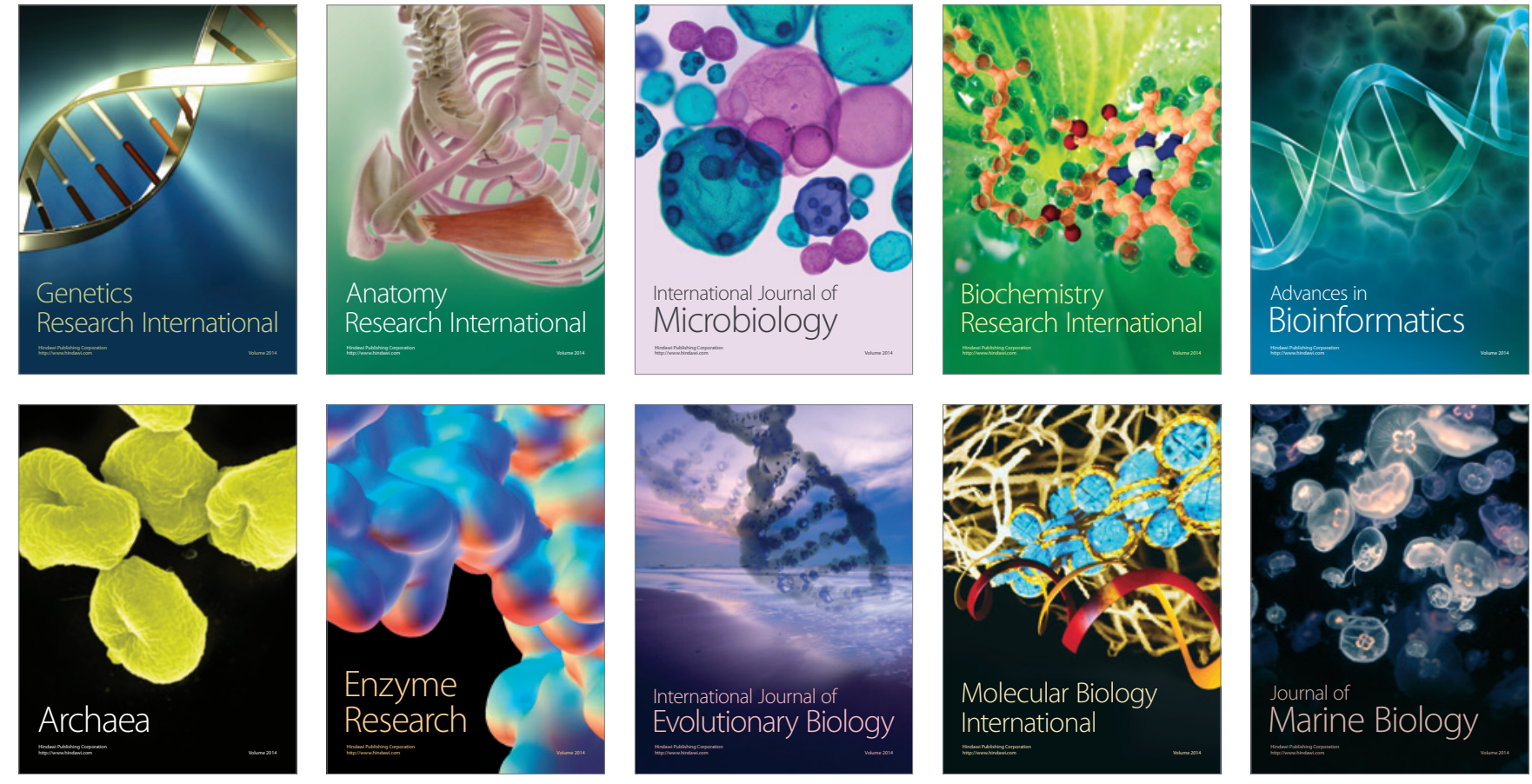